\title{
Original
}

\section{Application of The bFGF and $\beta$-TCP Complex to Peri-Implant Bone Defects in Dogs}

\author{
Masashi Takagi, Keiichi Kanayama, Keisuke Mukai, Hirotsugu Morinaga, Atsushi Shibatsuji and Toshiaki Shibutani
}

Department of Periodontology, Division of Oral Infections and Health Science, Asahi University School of Dentistry, Gifu, Japan (Accepted for publication, March 12, 2020)

\begin{abstract}
A bone substitute, beta-tricalcium phosphate, was combined with a basic fibroblast growth factor to create a fibroblast growth factor/tricalcium phosphate complex. This complex was then implanted into canine mandibles to evaluate the usefulness of this treatment in bony defects around dental implants. Four beagles received implants that were installed into fresh extraction sockets of third and fourth mandibular premolars. All surgical procedures were performed under general anesthesia with intravenous sodium thiopental. Gingival incisions were performed mesially from the second premolar and distally to molars. Full-thickness buccal and lingual flaps were raised. After vertical interradicular sectioning, each root was carefully elevated and then gently extracted. Buccal bone defects adjacent to extraction sockets with a height of $7 \mathrm{~mm}$ and 3 $\mathrm{mm}$ in the mesio-distal direction were created on third and fourth premolars, respectively, using round burs with sterile saline irrigation. These defects were then assigned to the control, fibroblast growth factor, tricalcium phosphate complex, or fibroblast growth factor/tricalcium phosphate group. Animals were sacrificed 4 weeks after surgery. Tissue blocks were fixed in $4 \%$ paraformaldehyde and then scanned with micro-computed tomography before sectioning for light microscopy. New bone height and bone mineral density in sites were significantly larger in tricalcium phosphate and fibroblast growth factor than in tricalcium phosphate groups. New bone height was also significantly larger in tricalcium phosphate and fibroblast growth factor/tricalcium phosphate groups than in fibroblast growth factor and control groups. These results indicate that fibroblast growth factor/tricalcium phosphate complex is useful as a growth factor carrier and scaffold for bone augmentation around dental implants.
\end{abstract}

Key words: basic Fibroblast growth factor, beta-Tricalcium phosphate, Bone regeneration, Dental implant

\section{Introduction}

Dental implants are increasingly being selected as a functional recovery procedure after tooth extraction attributed to periodontal disease. However, implants are not applicable in some cases due to large bone defects and irregular bone morphology. When the fixture is implanted, part of the implant body may be exposed. Various regenerative therapies have been reported for the purpose of bone regeneration in defects.

The effectiveness of beta-tricalcium phosphate $(\beta-\mathrm{TCP})$ has recently been reported as a bone substitute material ${ }^{1)}$. However, $\beta$-TCP has a chemical composition that is not present in living tissue. Thus, its biocompatibility is inferior to other bone substitute materials. $\beta$-TCP is considered to be absorbed in vivo ${ }^{2}$.

Furthermore, periodontal tissue regeneration therapy using basic fibroblast growth factor (bFGF) has been attempted ${ }^{3)}$. bFGF is expressed on undifferentiated mesenchyme, such as fibroblasts and vascular endothelial cells, via the FGF receptor. Previous studies reported its involvement in migration and differentiation of system cells and promotion of tissue repair, ${ }^{4}$. In animal experiments, local administration of bFGF to alveolar bone defects induced periodontal tissue regeneration. In addition, clinical trials reported its usefulness in human bone regeneration $^{6,7)}$. The bFGF used in clinical trials was hydroxypropyl cellulose (HPC). Although a gel-like complex was used, topical administration of

Correspondence to: Dr. Keiichi Kanayama, Department of Periodontology, Division of Oral Infections and Health Science, Asahi University School of Dentistry, 1851 Hozumi Mizuho, Gifu, 501-0296, Japan; Tel: +81-58-3291452; Fax: +81-58-329-1452; E-mail: k-k1@dent.asahi-u.ac.jp the gel-like complex alone was insufficient for severe bone defects, for which a scaffold for form retention is necessary and requires strength, osteoconductivity, biocompatibility, and bioabsorbability. Therefore, experiments using bFGF as a scaffold have been conducted, and promising findings have been reported for periodontal tissue regeneration ${ }^{8,9)}$.

In the present study, bone loss was induced around implants, and bone regeneration was examined by loading a complex containing $\beta$-TCP as a scaffold and adding bFGF.

\section{Materials and methods}

\section{Experimental materials}

The implant body was made of titanium and measured $3.0 \mathrm{~mm} \times 8.0$ mm (Integra-CPтM; Bicon Dental Implants, Boston, MA, USA). $\beta$-TCP (SynthoGraft ${ }^{\mathrm{TM}}$; Bicon Dental Implants) was used as granules with a porous structure of 500-1,000 $\mu \mathrm{m}$. Recombinant human bFGF (REGROTH ${ }^{\circledR}$; Kaken Pharmaceutical Co., Ltd., Tokyo, Japan) was used by dissolving $250 \mu \mathrm{g}$ in $1 \mathrm{ml}$ of physiological saline, and $1 \mathrm{ml}$ of bFGF solution was mixed with $\beta$-TCP granules and used as the bFGF- $\beta$-TCP complex.

\section{Animal experiments}

Four 18-month-old male beagles weighing 10-12 $\mathrm{kg}$ were used as experimental animals. The study was conducted in accordance with the Asahi University Animal Experimental Guidelines and the protocol was approved by the Asahi University Laboratory Animal Ethics Committee (approval number: 11-021). Experimental animals were intramuscularly 

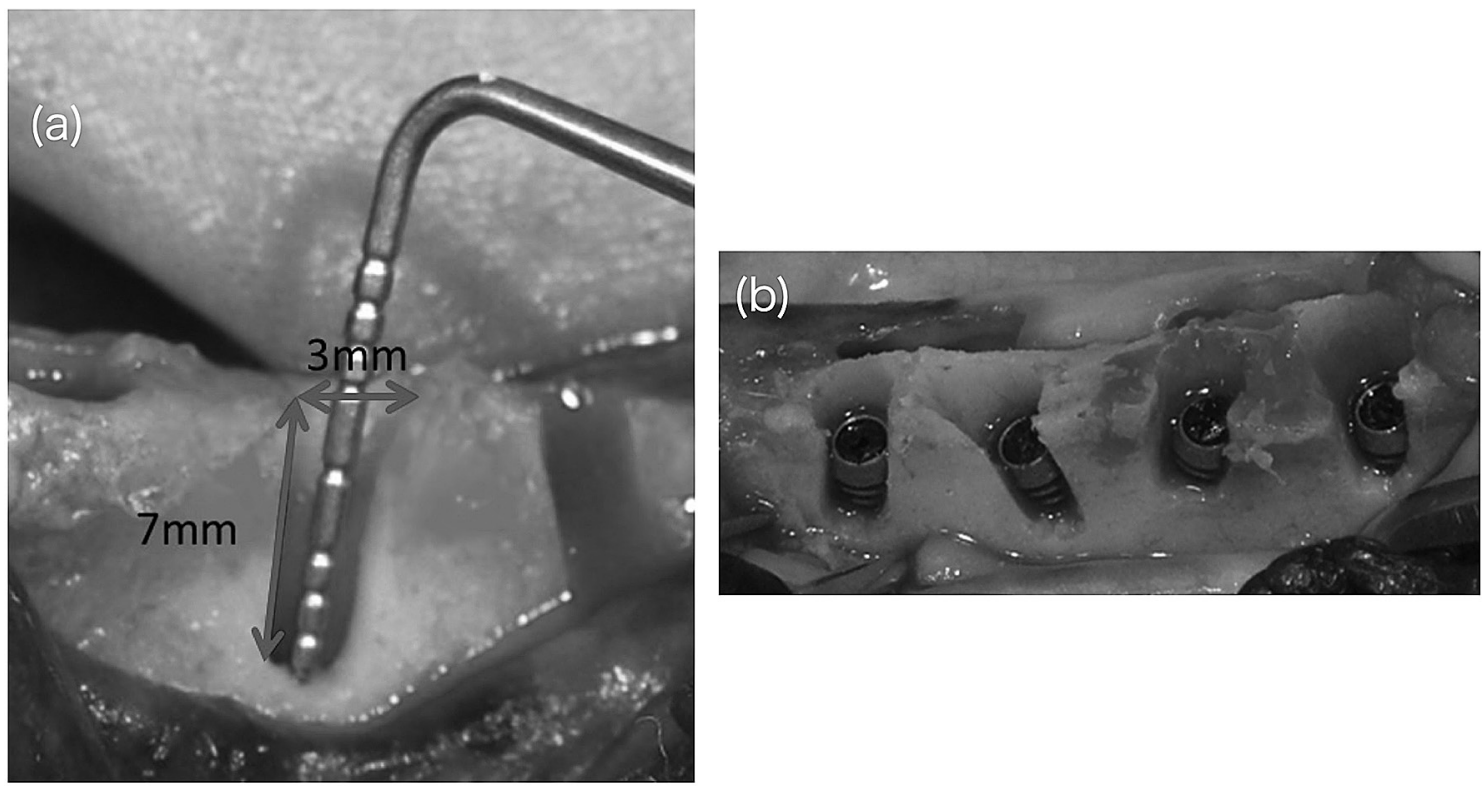

Figure 1. Photograph of the surgical procedure. (a) A bone defect is created on the buccal side of the extraction socket. (b) Implant bodies are implanted immediately after creation of bone defects.
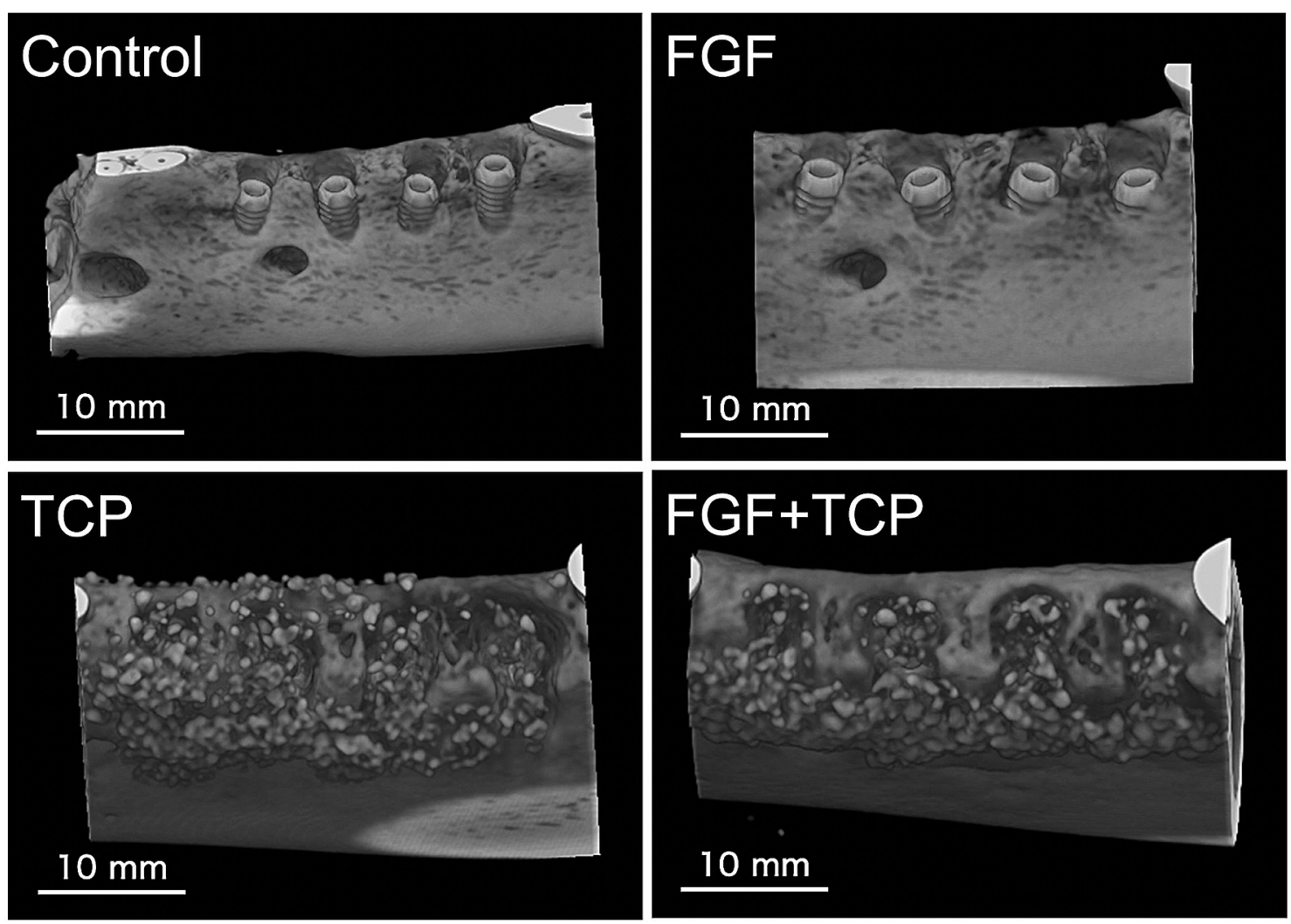

Figure 2. $\mu$-CT images 4 weeks after implantation.

injected with $0.1 \mathrm{ml} / \mathrm{kg}$ of xylazine hydrochloride ( $2 \%$ injectable solution of celactal 2; Bayer AG, Tokyo, Japan), and then intravenously injected with $0.5 \mathrm{ml} / \mathrm{kg}$ of sodium pentobarbital sodium (Somnopentyl®; Kyoritsu Seiyaku Corporation, Tokyo, Japan). General anesthesia was performed by an internal injection of $1.8 \mathrm{ml}$ of $2 \%$ lidocaine hydrochloride (xylocaine cartridge; Dentsply-Sankin, Tokyo, Japan) containing
1/100,000 epinephrine into third and fourth premolars on the lower jaw side of the treatment site ( $\mathrm{P} 3$ and $\mathrm{P} 4)$. After local anesthesia, gingival crevice incisions were made with a No. $15 \mathrm{c}$ blade and the gingiva was peeled off with a mucosal stripper using an all-layer valve. P3 and P4 were extracted. A pilot drill was used to create a hole in the fossa with a depth of $11.0 \mathrm{~mm}$. After enlarging the diameter to $3.0 \mathrm{~mm}$ with a latch 


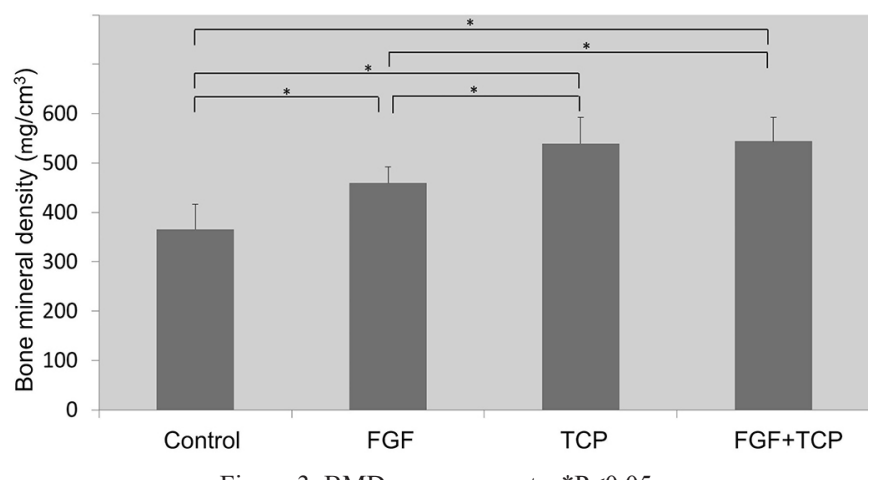

Figure 3. BMD measurements. ${ }^{*} \mathrm{P}<0.05$

reamer, bone defects were prepared using a tungsten carbide bur under water injection such that the buccal bone measured $3.0 \mathrm{~mm}$ in the mesio-distal direction and $7.0 \mathrm{~mm}$ in length (Fig. 1a).

Fixtures were immediately implanted $(n=8)$ (Fig. 1b). The healing plug was cut at the bone edge. Four groups were evaluated and compared: bFGF and $\beta$-TCP treatment (hereinafter referred to as FGF+TCP group), bFGF only treatment (FGF group), $\beta$-TCP only treatment (TCP group), and untreated (control group). Gingival flaps were then sutured with nylon thread (Soft Letch ${ }^{\circledR}$; GC Tokyo, Japan).

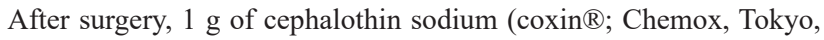
Japan) was intramuscularly injected once daily for 3 days. Four weeks after the observation period, animals were euthanized by excessive administration of sodium pentobarbital. Mandibles were removed and fixed in $4 \%$ paraformaldehyde (PFA) for 1 week.

\section{Micro-computed tomography analysis}

Samples were fixed in 4\% PFA and subjected to micro-computed tomography $(\mu-\mathrm{CT})$ with the following settings: tube voltage of $90 \mathrm{KV}$, tube current of $89 \mathrm{~mA}$, and magnification of $2.5 \times$ using a $\mu$-CT apparatus (Scanmate-RB 090 SS 150; Comscan, Tokyo, Japan). Bone mineral density (BMD) was measured with three-dimensional image analysis software (3D-BON; RATOC, Tokyo, Japan). The measurement range was from the defect bottom, i.e., the bone margin at the center of the implant, to $7 \mathrm{~mm}$ below the bone margin in the vertical direction, the implant diameter in the horizontal direction, and from the bone outside the defect bottom to the implant as the height.
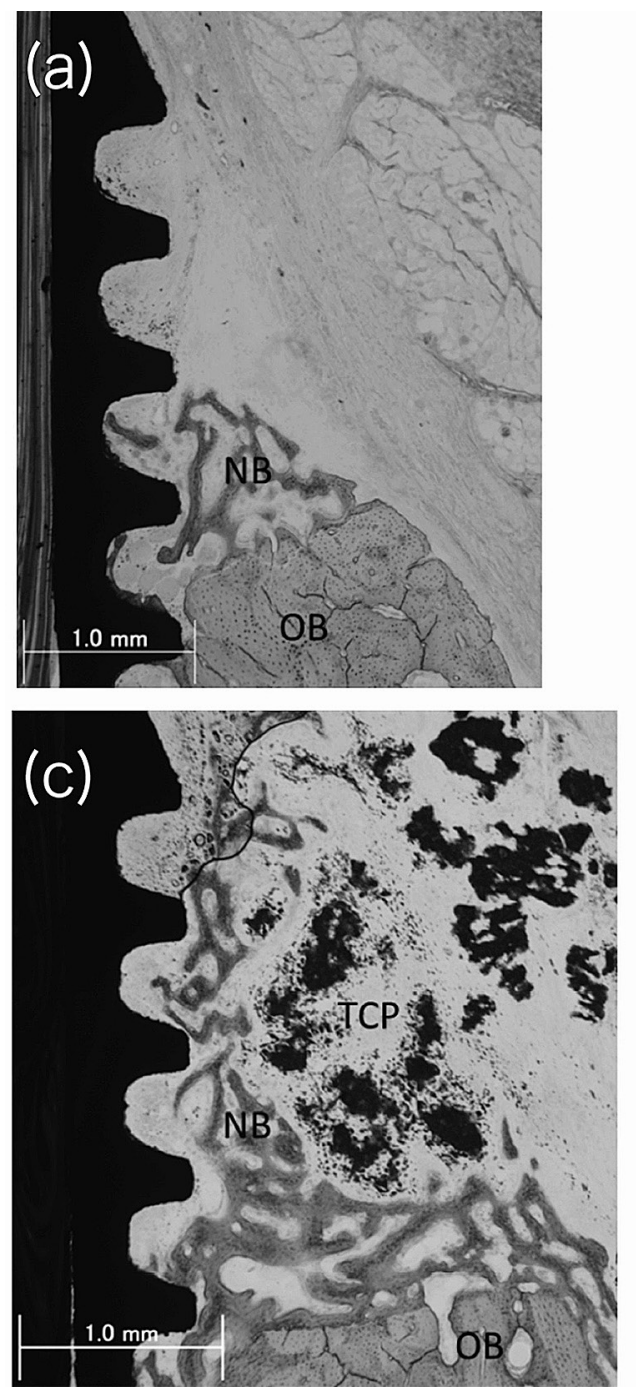
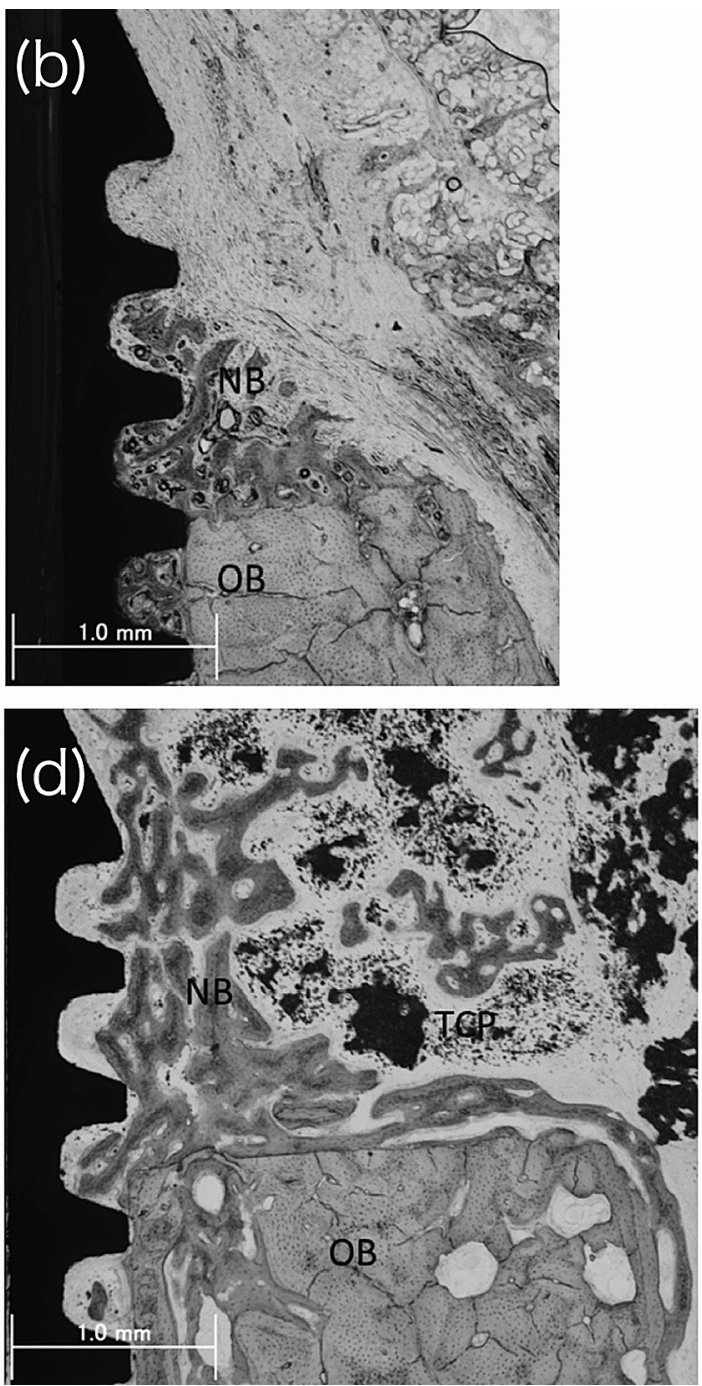

Figure 4. Hard tissue slices and staining with toluidine blue staining. (a) Histology of the control group. NB: new bone, OB: original bone. (b) Histology of the FGF group. (c) Histology of the TCP group. (d) Histology of the FGF+TCP group. 


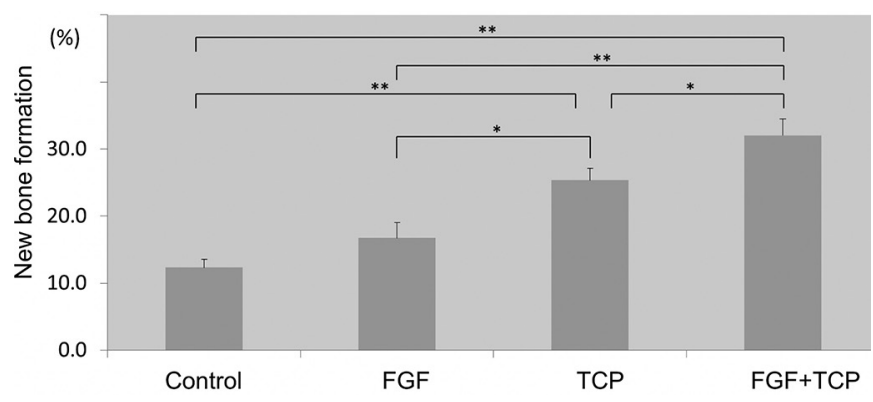

Figure 5. Measurement results for new bone mass. ${ }^{*} \mathrm{P}<0.05,{ }^{*} * \mathrm{P}<0.01$.

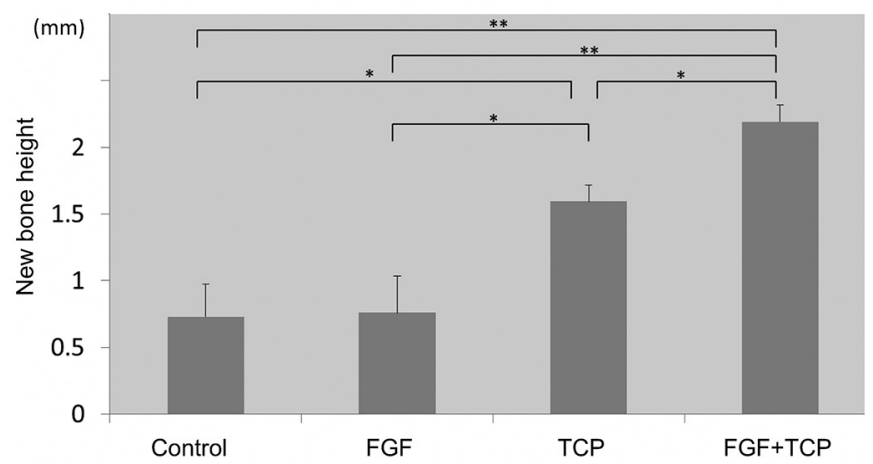

Figure 6. Measurement results for new bone height. $* \mathrm{P}<0.05, * * \mathrm{P}<0.01$.

\section{Preparation and observation of tissue samples}

Fixed samples were embedded in monomethyl methacrylate resin to prepare polished buccolingual sections with a thickness of $30 \mathrm{~mm}$ at the implant center. Sections were stained with toluidine blue and observed under an optical microscope.

\section{Measurement of new bone mass and height}

Tissue morphometry was performed on stained sections in the control, FGF, TCP, and FGF+TCP groups using image processing software (ImageJ, National Institutes of Health, Bethesda, MD, USA). Ratios of newly formed bone were determined from outside of the buccal bone on the defect bottom to outside of the implant in the horizontal direction and from the defect bottom to the upper end of the implant in the longitudinal direction. New bone height indicated the height of new bone adjacent to the fixture from the defect bottom.

\section{Statistical analysis}

To analyze the significance of differences between BMD, new bone mass, and new bone height, multiple comparison tests were performed using the Bonferroni-Dunn method. P values $<0.05$ were considered statistically significant. An independent statistician reviewed the statistical methodology, results and conclusions of the study.

\section{Results}

\section{Clinical observations}

No significant clinical changes were observed in experimental animals throughout the entire study period, and no detachment of the implant body was noted. Redness and slight swelling were detected 1 week postoperatively but subsequently decreased at 4 weeks. Animals showed normal gingival morphology, and no significant differences were noted among groups.

\section{$\boldsymbol{\mu}$-CT findings}

$\mu$-CT images 4 weeks after implantation confirmed the presence of residual $\beta$-TCP granules in TCP and FGF+TCP groups (Fig. 2). BMD was $365.4 \pm 50.4 \mathrm{mg} / \mathrm{kg}$ (mean \pm standard deviation) in the control group, $459.5 \pm 32.1 \mathrm{mg} / \mathrm{kg}$ in the FGF group, $539.4 \pm 52.7 \mathrm{mg} / \mathrm{kg}$ in the TCP group, and $536.0 \pm 48.0 \mathrm{mg} / \mathrm{kg}$ in the FGF+TCP group. Significant differences were observed between the control group and the FGF, TCP, and $\mathrm{FGF}+\mathrm{TCP}$ groups (all $\mathrm{P}<0.05$ ). No significant differences were noted between the TCP and FGF+TCP groups (Fig. 3).

\section{Histological observations}

A small amount of new bone continuing from existing bone was not detected in the control group. However, bone with wide trabeculae was observed, and contact with the implant body was only partially noted (Fig. 4a). In the FGF group, compact, small, new bone was observed between trabeculae, inflammatory cells were not detected, and contact with the implant body was noted in the proximity of existing bone (Fig. $4 b)$. For the TCP group, a large number of $\beta$-TCP granules were found in the defect. New bone formed between $\beta$-TCP granules and the fixture surface. New bone had wide trabeculae with prominent bone formation on the side of the fixture and weak formation on the buccal side, and some $\beta$-TCP granules remained (Fig. 4c). Finally, in the FGF+TCP group, prominent formation of new bone was observed in the defect. New bone formed around the upper end of the implant, with narrow, dense, wide bones observed between trabeculae. Although residual $\beta$-TCP granules were observed, inflammatory cell infiltration was not detected (Fig. 4d).

The amount of new bone formed was $12.3 \pm 1.2 \%$ in the control group, $16.7 \pm 2.3 \%$ in the FGF group, $25.4 \pm 1.8 \%$ in the TCP group, and $32.0 \pm 2.4 \%$ in the FGF+TCP group. Comparisons among groups revealed a significant difference between TCP and FGF+TCP groups $(\mathrm{P}<0.01)$. The amount of new bone formed was significantly greater in the FGF+TCP group than in TCP $(\mathrm{P}<0.05)$, control, and FGF groups $(\mathrm{P}<0.01)$. No significant differences were observed between the control and FGF groups (Fig. 5).

New bone height was $0.7 \pm 0.2 \mathrm{~mm}$ (mean \pm standard deviation) in the control group, $0.7 \pm 0.2 \mathrm{~mm}$ in the FGF group, $1.5 \pm 0.1 \mathrm{~mm}$ in the TCP group, and $2.1 \pm 0.1 \mathrm{~mm}$ in the FGF+TCP group. Significant differences were observed between the FGF group and TCP $(\mathrm{P}<0.05)$, FG$\mathrm{F}+\mathrm{TCP}(\mathrm{P}<0.01)$, and TCP groups $(\mathrm{P}<0.05)$. A significant difference was also noted between the TCP and FGF+TCP groups $(\mathrm{P}<0.05)$. No significant differences were found between the control and FGF groups (Fig. $6)$.

\section{Discussion}

A composite material comprising $\beta$-TCP and platelet-derived growth factor (PDGF) is currently used as a bone substitute material with cytokines. PDGF is released from platelets and endothelial cells at damaged sites and is present between fibroblasts. Previous studies reported its involvement in wound healing through migration and proliferation of mesenchymal cells ${ }^{10}$, and it is also known to be useful for periodontal tissue regeneration ${ }^{11,12)}$. Moreover, bone regeneration in bone defects around the implant was more greatly enhanced with PDGF than with $\beta-\mathrm{TCP}^{13)}$.

In this study, a complex in which bFGF was added as a scaffold was used for implants surrounding bone defects. At postoperative 4 weeks, residual $\beta$-TCP granules were confirmed in $\mu$-CT images. BMD was not significantly different between the TCP and FGF+TCP groups. Anzai et al. ${ }^{14)}$ evaluated bone mineral content using bFGF- $\beta$-TCP composite ma- 
terial. No significant differences were observed at 3 weeks, while a significant increase was noted at 6 weeks. The discrepancy in results between our and the previous study may be explained by differences in experimental periods. However, there were some technical difficulties in discriminating between new bone surrounding $\beta$-TCP granules and residual $\beta$-TCP in the $\mu$-CT analysis due to the artifact of the implanted body.

Histological findings showed prominent new bone formation on the implant fixture and residual $\beta$-TCP granules on the buccal side in the TCP and FGF+TCP groups. $\beta$-TCP is considered to have a high osteoconductive capacity ${ }^{15)}$. Shirakata et al. ${ }^{16)}$ showed that bFGF-HPC and PDGF- $\beta$ TCP composites induced similar periodontal tissue regeneration in animal experiments. Furthermore, residual $\beta$-TCP was suggested to inhibit bone regeneration. Residual $\beta$-TCP was previously reported to delay bone regeneration ${ }^{17)}$. However, Oi et al. ${ }^{18)}$ found that porous $\beta$-TCP was absorbed by osteoclasts and ultimately replaced with bone. In the present study, dense bone formation was observed when bFGF was added, but new bone formation in the FGF group was not similar to that in TCP and FGF+TCP groups. This finding may be attributed to bFGF being dissolved in saline and used in a liquid state, which remained in the defect for a short period of time. Moreover, new bone mass and height were significantly greater in the TCP and FGF+TCP groups than in the FGF and control groups. This result suggested that $\beta$-TCP retains the defective part and its osteoconductive ability. Furthermore, the significant difference observed between the TCP and FGF+TCP groups suggested that $\beta$-TCP effectively acts as a scaffold for bFGF. Bone regeneration started at an early stage in the bone defect around the implant body. However, in the present study, the effects of residual $\beta$-TCP were not followed up, and therefore should be examined in the future.

\section{Acknowledgements}

This work was supported by JSPS KAKENHI Grant Numbers 15K20572 and 19K10232.

\section{Conflict of interest}

The authors have no conflicts of interest directly relevant to the content of this article.

\section{References}

1. Stavropoulos A, Windisch P, Szendröi-Kiss D, Peter R, Gera I and Sculean A. Clinical and histologic evaluation of granular beta-tricalcium phosphate for the treatment of human intrabony periodontal defects: A report on five cases. J Periodontol 81: 325-334, 2010

2. Nagayama M, Takeuchi H and Doi Y. Comparison of carbonate apatite and $\beta$-tricalcium phosphate (resorbable calcium phosphates) implanted subcutaneously into the back of rats. Dent Mater J 25: 219 225, 2006

3. Murakami S, Takayama S, Kitamura M, Shimabukuro Y, Yanag K, Ikezawa K, Saho T, Nozaki T and Okada H. Recombinant human basic fibroblast growth factor (bFGF) stimulates periodontal regeneration in class II furcation defects created in beagle dogs. J Periodont Res 38: 97-103, 2003

4. Barrientos S, Stojadinovic O, Golinko MS, Brem H and Tomic-Canic M. Growth factors and cytokines in wound healing. Wound Rep Reg 16: 585-601, 2008

5. Bikfalvi A, Klein S, Pintucci G and Rifkin DB. Biological roles of fibroblast growth factor-2. Endocr Rev 18: 26-45, 2009

6. Kitamura M, Akamatsu M, Machigashira M, Hara Y, Sakagami R,
Hirofuji T, Hamachi T, Maeda K, Yokota M, Kido J, Nagata T, Kurihara H, Takashiba S, Shibutani T, Fukuda M, Noguchi T, Yamazaki $\mathrm{K}$, Yoshie H, Ioroi K, Arai T, Nakagawa T, Ito K, Oda S, Izumi Y, Ogata Y, Yamada S, Shimauchi H, Kunimatsu K, Kawanami M, Fujii T, Furuichi Y, Furuuchi T, Sasano T, Imai E, Omae M, Yamada S, Watanuki M and Murakami S. FGF-2 stimulates periodontal regeneration : results of a multi-center randomized clinical Trial. J Dent Res 90: 35-40, 2011

7. Kitamura M, Nakashima K, Kowashi Y, Fujii T, Shimauchi H, Sasano T, Furuuchi T, Fukuda M, Noguchi T, Shibutani T, Iwayama Y, Takashiba S, Kurihara H, Ninomiya M, Kido J, Nagata T, Hamachi T, Maeda K, Hara Y, Izumi Y, Hirofuji T, Imai E, Omae M, Watanuki $\mathrm{M}$ and Murakami S. Periodontal tissue regeneration using fibroblast growth factor-2: randomized controlled phase II clinical trial. Plos One 3: e2611, 2008

8. Ishii Y, Fujita T, Okubo N, Ota M, Yamada S and Saito A. Effect of basic fibroblast growth factor (FGF-2) in combination with beta tricalcium phosphate on root coverage in dog. Acta Odontol Scand 71: 325-332, 2013

9. Cochran DL, Oh TJ, Mills MP, Clem DS, McClain PK, Schallhorn RA, McGuire MK, Scheyer ET, Giannobile WV, Reddy MS, Abou-Arraj RV, Vassilopoulos PJ, Genco RJ, Geurs NC and Takemura A. A randomized clinical trial evaluating rh-FGF-2/ $\beta-\mathrm{TCP}$ in periodontal defects. J Dent Res 95: 523-30, 2016

10. Reuterdahi C, Sundberg C, Rubin k, Funa K and Gerdin B. Tissue localization of beta receptors for platelet-derived growth factor and platelet-derived growth factor B chain during wound repair in humans. J Clin Invest 91: 2065-2075, 1993

11. Nevins M, Camelo M, Nevins ML, Schenk RK and Lynch SE. Periodontal regeneration in humans using recombinant human platelet-derived growth factor-BB (rhPDGF-BB) and allogenic bone. J Periodontol 74: 1282-1292, 2003

12. Sarment DP, Cooke JW, Miller SE, Jin Q, McGuire MK, Kao RT, McClain PK, McAllister BS, Lynch SE and Giannobile WV. Effect of rhPDGF-BB on bone turnover during periodontal repair. J Clin Periodontol 33: 135-140, 2006

13. Choo T, Marino V and Bartold PM. Effect of PDGF-BB and beta-tricalcium phosphate $(\beta-\mathrm{TCP})$ on bone formation around dental implants: a pilot study in sheep. Clin Oral Impl Res 24: 158-166, 2013

14. Anzai J, Kitamura M, Nozaki T, Nagayasu T, Terashima A, Asano T and Murakami S. Effects of concomitant use of fibroblast growth factor (FGF)-2 with beta-tricalcium phosphate ( $\beta$-TCP) on the beagle dog 1-wall periodontal defect model. B B Res Commun 403: 345-350, 2010

15. Yuan H, Kurashina K, de Bruijn JD, Li Y, de Groot K and Zhang X. A preliminary study on osteoinduction of two kinds of calcium phosphate ceramics. Biomaterials 20: 1799-1806, 1999

16. Shirakata Y, Taniyama K, Yoshimoto T, Miyamoto M, Takeuchi N, Matsuyama $\mathrm{T}$ and Noguchi K. Regenerative effect of basic fibroblast growth factor on periodontal healing in two-wall intrabony defects in dogs. J Clin Periodontol 37: 374-381, 2010

17. Araujo M, Liljenberg B and Lindhe J. $\beta$-tricalcium phosphate in the early phase of socket healing: an experimental study in the dog. Clin Oral Impl Res 21: 445-454, 2010

18. Oi Y, Ota M, Yamamoto S, Shibukawa Y and Yamada S. $\beta$-tricalcium phosphate and basic fibroblast growth factor combination enhances periodontal regeneration in intrabony defects in dogs. Dent Mater J 28: 162-169, 2009 
J.Hard Tissue Biology Vol. 29(2): 105-110, 2020 